\title{
Stabilization of unstable steady states by variable delay feedback control
}

\author{
Aleksandar Gjurchinovsk'* and Viktor Urumov' \\ Department of Physics, Faculty of Natural Sciences and Mathematics, \\ Sts. Cyril and Methodius University, P. O. Box 162, 1000 Skopje, Macedonia
}

(Dated: May 22, 2008)

\begin{abstract}
We report on a dramatic improvement of the performance of the classical time-delayed autosynchronization method (TDAS) to control unstable steady states, by applying a time-varying delay in the TDAS control scheme in a form of a deterministic or stochastic delay-modulation in a fixed interval around a nominal value $T_{0}$. The successfulness of this variable delay feedback control (VDFC) is illustrated by a numerical control simulation of the Lorenz and Rössler systems using three different types of time-delay modulations: a sawtooth wave, a sine wave, and a uniform random distribution. We perform a comparative analysis between the VDFC method and the standard TDAS method for a sawtooth-wave modulation by analytically determining the domains of control for the generic case of an unstable fixed point of focus type.
\end{abstract}

PACS numbers: $05.45 . \mathrm{Gg}, 02.30 . \mathrm{Ks}$

\section{INTRODUCTION}

The idea of controlling chaos has been initiated by Ott, Grebogy and Yorke in a seminal paper proposing a routine to stabilize unstable orbits embedded in chaotic attractors [1]. This control scheme, now recognized as the OGY method, is taking advantage of the ergodicity of the system by applying a small perturbation to a suitably chosen control parameter when the trajectory of the system is sufficiently close to the stable manifold of the local linear system. Since the OGY paper, the control of chaos has become a topic of broad interest among scientists, both theoretically and experimentally [2, 3, 4, 5]. Although different control schemes were developed, most of them are relying on the general concept underlying OGY scheme - stabilizing an orbit embedded into the chaotic system.

An alternative and very practical implementation of the OGY idea is the time-delayed autosynchronization (TDAS) introduced by Pyragas in 1992 [6, 7, 8]. It is based on a continuous feedback applied in the form of a control force proportional to the difference of the current state of the system at time $t$ and its counterpart at some instant $t-T$ in the past. If the delay time $T$ is set equal to the period of the unstable periodic orbit (UPO) whose stabilization is required, the control signal vanishes when the system is on that orbit, so that the extracted orbit remains a solution of the equations describing the dynamics of the original system. In this sense, the Pyragas method is non-invasive.

A generalization of Pyragas method was suggested by Socolar, Sukow and Gauthier in 1994 [9, 10], where the feedback signal was taken in the form of a geometric sum (extended time-delayed autosynchronization - ETDAS), or a mean value of a finite number of delay terms (N

\footnotetext{
*Electronic address: agjurcin@iunona.pmf.ukim.edu.mk
}

${ }^{\dagger}$ Electronic address: urumov@iunona.pmf.ukim.edu.mk time-delayed autosynchronization - NTDAS), each using information from many previous states of the system involving integer multiples of the delay time $T$. In spite of introducing an additional control parameter, ETDAS has been recognized as the most important modification of the TDAS, since it achieves stabilization of UPOs with a higher degree of instability.

Parallel to the efforts of stabilizing UPOs, the Pyragas' method and its various extensions were used to stabilize unstable steady states (USS) [11, 12, 13, 14, 15]. Among these is the multiple delay feedback control (MDFC) recently suggested by Albhorn and Parlitz 16, 17]. The scheme is a natural extension of ETDAS method, using two or more delayed feedback signals with incommensurate delay times. Although being more effective in comparison to TDAS and ETDAS, the MDFC has some drawback by introducing additional control parameters with every additional feedback signal.

To improve the performance of TDAS in controlling unstable steady states, with this paper we suggest a variable delay feedback control (VDFC), where the delay time $T$ is not kept constant during the control process, but it is modulated in time in a suitably chosen way. We demonstrate the VDFC method for the paradigmatic Lorenz and Rössler systems, using three different types of modulation of the delay time $T(t)$ around a nominal value $T_{0}$ in a fixed interval $\left[T_{0}-\varepsilon, T_{0}+\varepsilon\right]$ determined by the control parameter $\varepsilon$. To make a comparison with the TDAS method, we perform a linear stability analysis of the VDFC for stabilizing a two-dimensional unstable steady state of a focus type for different values of the parameter $\varepsilon$. By using the same method of analysis, we calculated the parameter regions for successful VDFC stabilization of the unstable steady states in the threedimensional Lorenz and Rössler systems. We show that for $\varepsilon=0 \mathrm{VDFC}$ is reduced to TDAS, and for $\varepsilon>0$ the domain of control is drastically enlarged with respect to TDAS. The correctness of the stability analysis is supported by computer simulations. 


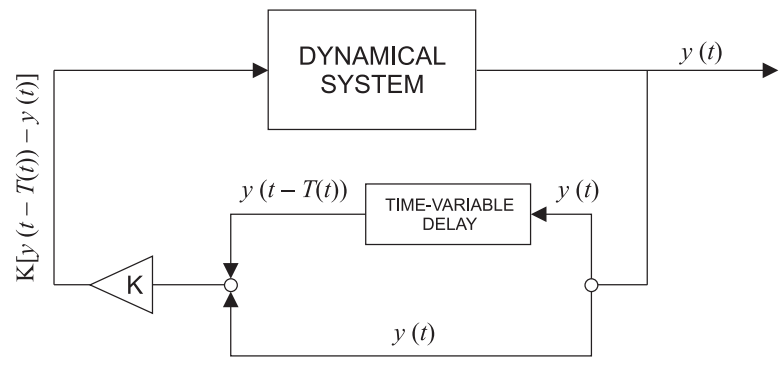

FIG. 1: Schematic of the variable delay feedback control

\section{VARIABLE DELAY FEEDBACK CONTROL}

The Pyragas' method assumes a dynamical system having at least one scalar variable $y(t)$ accessible for measurements, and an input channel through which the external force $F(t)$ is fed back to the system. The standard TDAS control scheme reads:

$$
\begin{aligned}
\frac{d}{d t} \mathbf{x}(t) & =\mathbf{Q}(y(t), \mathbf{x}(t)), \\
\frac{d}{d t} y(t) & =P(y(t), \mathbf{x}(t))+F(t),
\end{aligned}
$$

where

$$
F(t)=K[y(t-T)-y(t)]
$$

is the external feedback force which is reinjected into the system through the $y$-channel as the difference between the signal $y(t)$ and the delayed signal $y(t-T)$ multiplied by a constant weighting factor $K$. The remaining variables of the system are represented by the vector $\mathbf{x}$, and $P$ and $\mathbf{Q}$ are nonlinear functions. The scheme involves only two control parameters - the delay time $T$ and the control gain $K$. For an effective stabilization of USSs, the delay time $T$ is related to the intrinsic characteristic timescale given by the imaginary part of the stationary point eigenvalue [1].

The external feedback force (3) in the case of VDFC reads:

$$
F(t)=K[y(t-T(t))-y(t)],
$$

where $T(t)$ is the time-dependent delay time. The schematic of VDFC is shown in Fig. 1.

To illustrate the VDFC method for stabilizing unstable steady states, we will use the Lorenz system [18]:

$$
\begin{aligned}
& \frac{d}{d t} x(t)=-10 x(t)+10 y(t), \\
& \frac{d}{d t} y(t)=28 x(t)-y(t)-x(t) z(t)+F(t), \\
& \frac{d}{d t} z(t)=-8 / 3 z(t)+x(t) y(t),
\end{aligned}
$$

assuming that the output variable is $y(t)$ and that the external feedback signal (4) perturbs only the second equation of the system. The trajectory of the unperturbed system $(K=0)$ is shown in panel (a) of Fig. 2, where the appearance of the chaotic attractor becomes evident.

The Lorenz system has three equilibrium points, one at the origin $C_{0}(0,0,0)$, and two positioned symmetrically with respect to the $z$-axis at $C^{+}(6 \sqrt{2}, 6 \sqrt{2}, 27)$ and $C^{-}(-6 \sqrt{2},-6 \sqrt{2}, 27)$. Linearizing the Lorenz equations around the equilibrium points reveals the form of their stability. The eigenvalues of the unperturbed fixed point $C_{0}$ at the origin are $\lambda\left(C_{0}\right) \approx\{-22.83,11.83,-2.67\}$ indicating an unstable three-dimensional saddle with one positive and two negative real eigenvalues. The other two fixed points have the same type of stability given by their common set of eigenvalues $\lambda\left(C^{ \pm}\right) \approx\{-13.85,0.09+$ $10.19 i, 0.09-10.19 i\}$. They are unstable fixed points of the focus type, having one negative real eigenvalue and complex-conjugate pair of eigenvalues with positive real part.

Panels (b)-(g) of Fig. 2 depict the dynamics of the variable $x(t)$ and the feedback signal $F(t)$ obtained from a computer simulation of the VDFC-controlled Lorenz system (5)-(7), indicating a successful control of the fixed points $C^{ \pm}$by using three different types of modulation of the delay time $T(t)$ in an $\varepsilon$-neighbourhood around a fixed value $T_{0}$. The initial conditions of the system were chosen $x(0)=2, y(0)=3$ and $z(0)=4$. The control was activated at $t=300$. Panels (b) $-(\mathrm{c})$ correspond to time-modulation in a form of a sawtooth wave with an inverse period $a$ :

$$
T(t)=T_{0}+\varepsilon[2(\text { at } \bmod 1)-1],
$$

panels (d)-(e) represent the time-series for a uniform random distribution of the delay times $T(t)$ in an interval $\left[T_{0}-\varepsilon, T_{0}+\varepsilon\right]$ :

$$
T(t)=T_{0}+\varepsilon \text { Random }[-1,1],
$$

and panels (f)-(g) are related to periodic sine-wave modulation:

$$
T(t)=T_{0}+\varepsilon \sin (a t) .
$$

In each case, the control parameters were chosen as $K=0.5, T_{0}=30$ and $\varepsilon=1$, with $a=10$ for the sawtooth and sine waves. We note that the values for the control parameter $\varepsilon$ are limited to the interval $0 \leq \varepsilon \leq T_{0}$. In panels (c), (e) and (g) we see that the feedback signal $F(t)$ vanishes when the stabilization of the fixed point is achieved, suggesting noninvasiveness of VDFC. The noninvasiveness of the control method follows from the form of the control force in Eq. (4), since $y(t-T(t))=y(t)$ if the fixed point is stabilized. We notice that the saddle point $C_{0}$ at the origin cannot be stabilized with the VDFC scheme, obeying the odd-number limitation theorem that a Pyragas-type control in its standard form is limited to fixed points characterized by a finite torsion, which do not have an odd number of positive real eigenvalues [19, 20, 21, 22]. The fixed points $C^{ \pm}$do not have positive real eigenvalues, and they can be stabilized with 

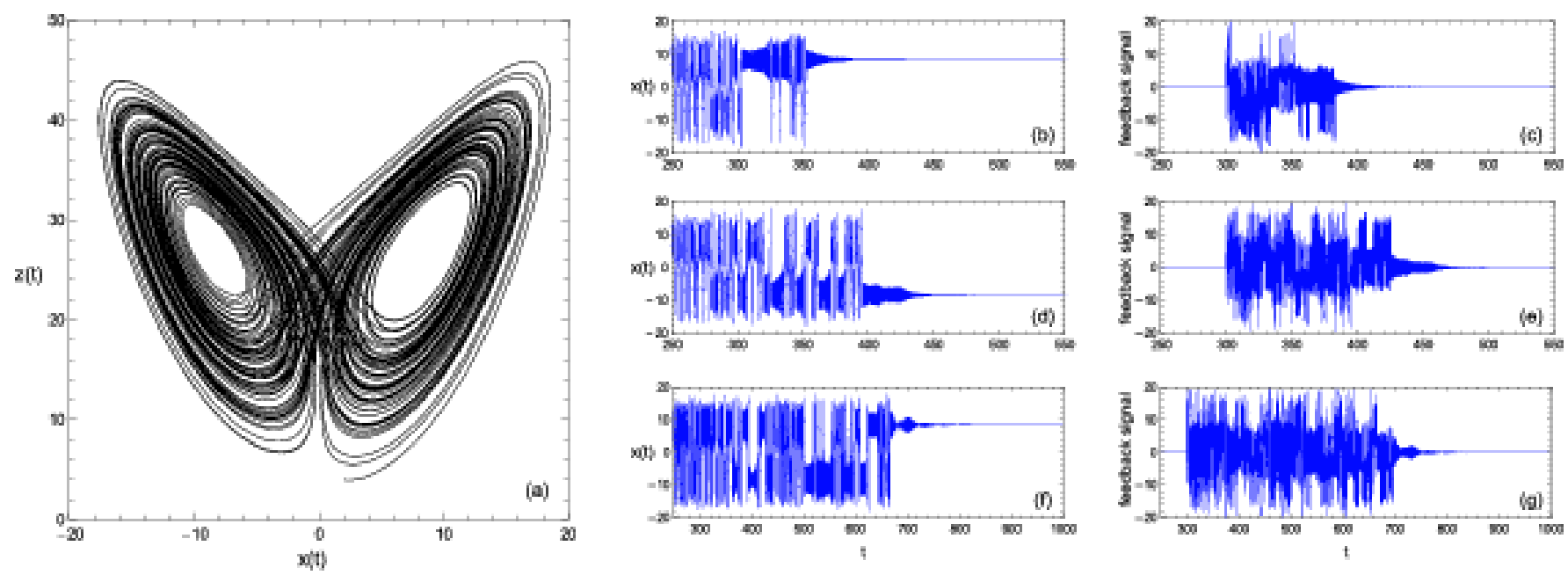

FIG. 2: (Color online) A simulation of variable delay feedback control in the Lorenz system (5)-(7) using three different modulations of the delay time. (a) The Lorenz attractor of the unperturbed system; (b),(c) The time-series of the variable $x(t)$ and the feedback signal $F(t)$ of the controlled system corresponding to a sawtooth-wave modulation with $a=10$, indicating a successful control of the unstable fixed point at $C^{+} ;(\mathrm{d}),(\mathrm{e})$ The corresponding time-series for a uniform random modulation controlling the unstable point at $C^{-} ;(\mathrm{f}),(\mathrm{g})$ The time-series for a sine-wave modulation $(a=10)$, stabilizing the unstable point at $C^{+}$. In each case, the initial conditions were: $x(0)=2, y(0)=3$ and $z(0)=4$, the control parameters were: $K=0.5$, $T_{0}=30$ and $\varepsilon=1$, and the control was activated at $t=300$. Note the different scales on the $t$-axis on different panels.

the proposed version of VDFC, as indicated in Fig. 2. Since the fixed points $C^{+}$and $C^{-}$have identical eigenvalues, the Lorenz system can be stabilized to either of these states, depending on the initial conditions. In the case of uniform random modulation (9), the preference of control toward either $C^{+}$or $C^{-}$will also depend on the random number sequence used in the modulation. In the latter case, we noticed that for identical initial conditions and different uniform random sequences, the preference of stabilization of the system varies between $C^{+}$and $C^{-}$. Also, keeping the random sequence fixed and varying the starting position of the sequence influences the preference of the control. These observations could be related to the structure of the basins of attraction for the stabilized steady states $C^{ \pm}$. [23]:

The simulation was repeated for the Rössler system

$$
\begin{aligned}
& \frac{d}{d t} x(t)=-y(t)-z(t), \\
& \frac{d}{d t} y(t)=x(t)+0.2 y(t)+F(t), \\
& \frac{d}{d t} z(t)=0.2+(x(t)-5.7) z(t),
\end{aligned}
$$

with the feedback force $F(t)$ as given in Eq. (4), using the same types of time modulation of the delay time $T(t)$. The unperturbed Rössler system has two unstable fixed points of the focus type at $C_{1}(0.007,-0.035,0.035)$ and $C_{2}(5.693,-28.465,28.465)$ with corresponding eigenvalues $\lambda\left(C_{1}\right) \approx\{-5.687,0.097+0.995 i, 0.097-0.995 i\}$ and $\lambda\left(C_{2}\right) \approx\left\{0.192,-4.596 \cdot 10^{-6}+5.428 i,-4.596 \cdot 10^{-6}-\right.$ $5.428 i\}$. The stabilization has been achieved only for the fixed point $C_{1}$, since it doesn't possess positive real eigenvalues, whereas the fixed point $C_{2}$ has one, and therefore, cannot be stabilized with the present version of VDFC. We omit a detailed discussion on the stabilization process and the simulation figures describing the dynamics of the control, since they concur to the ones for the Lorenz system.

\section{THE MECHANISM OF VDFC}

To provide an insight into the mechanism of the VDFC method and to reveal its superiority over the classical TDAS, we will initially consider a two-dimensional nonlinear system of first-order differential equations:

$$
\frac{d}{d t} \mathbf{x}(t)=\mathbf{f}[\mathbf{x}(t)]
$$

with $\mathbf{x}(t)=\operatorname{Col}[x(t), y(t)]$ being the state column vector of the system, and $\mathbf{f}$ the field vector describing the dynamics of the system. The stability of a particular critical point $\overline{\mathbf{x}}$ can be determined by linearizing the vector field $\mathbf{f}$ around $\overline{\mathbf{x}}$. We assume that the system (14) has undergone a coordinate transformation, such that the critical point $\overline{\mathbf{x}}$ is at the origin $(\overline{\mathbf{x}}=\mathbf{0})$. We also assume that the critical point $\overline{\mathbf{x}}=\mathbf{0}$ is an unstable fixed point of focus type. Here we have taken into account the limitation of the original Pyragas scheme, suggesting a failure of the control method for a torsion-free situation, i. e. if the number of positive real eigenvalues of the unstable fixed point is odd. The linearized version of the system (14) 
in center manifold coordinates can be written as:

$$
\frac{d}{d t} \mathbf{x}(t)=\mathbf{A x}(t)
$$

where

$$
\mathbf{A}=\left(\begin{array}{cc}
\lambda & \omega \\
-\omega & \lambda
\end{array}\right)
$$

is the matrix that determines the dynamics of the unperturbed system. The matrix $\mathbf{A}$ has complex conjugate eigenvalues $\Lambda_{0}=\lambda \pm i \omega$, with $\lambda$ and $\omega$ positive real numbers, warranting an unstable focus at the origin.

To stabilize the unstable fixed point at the origin using VDFC, we will perturb the system (14) with an additional control force $\mathbf{F}(t)$ in the diagonal form:

$$
\mathbf{F}(t)=K\left(\begin{array}{c}
x(t-T(t))-x(t) \\
y(t-T(t))-y(t)
\end{array}\right),
$$

where $K$ is the feedback strength, and $T(t)$ is the timedependent delay time. The linearized system (15) now obtains the form:

$$
\frac{d}{d t} \mathbf{x}(t)=\mathbf{A x}(t)+\mathbf{F}(t)
$$

which, in the case when $T(t)$ is constant, reduces itself to the standard diagonal TDAS control scheme. In the following, we consider a modulated time-delay $T(t)$ around a nominal value $T_{0}$, in a form of a periodic sawtooth wave (8). In this case, the delay time $T(t)$ is uniformly distributed over the interval $\left[T_{0}-\varepsilon, T_{0}+\varepsilon\right]$. According to Michiels-Van Assche-Niculescu (MAN) theorem [24], the stability of (18) under the variable-delay control force (17) can be inferred from the stability of the analogous time-invariant system with a distributed delay:

$$
\frac{d}{d t} \mathbf{x}(t)=\mathbf{A} \mathbf{x}(t)+\widetilde{\mathbf{F}}(t)
$$

having a control force $\widetilde{\mathbf{F}}(t)$ in the form:

$$
\widetilde{\mathbf{F}}(t)=K\left(\begin{array}{c}
\frac{1}{2 \varepsilon} \int_{t-T_{0}-\varepsilon}^{t-T_{0}+\varepsilon} x(\theta) d \theta-x(t) \\
\frac{1}{2 \varepsilon} \int_{t-T_{0}-\varepsilon}^{t-T_{0}+\varepsilon} y(\theta) d \theta-y(t)
\end{array}\right) .
$$

The theorem asserts that if the comparison system (19)(20) is asymptotically stable, then the original system (17) - (18) is globally uniformly asymptotically stable for sufficiently large values of the inverse period $a$ of the modulation. We note that in the case of a non-uniform deterministic distribution of $T(t)$ in the interval $\left[T_{0}-\right.$ $\left.\varepsilon, T_{0}+\varepsilon\right]$, an additional multiplicative factor will appear in the integrals in Eq. (20) depending on the form of $T(t)$, due to the non-constant weight of the distribution.
Using the ansatz $\mathbf{x}(t) \sim \exp (\bar{\Lambda} t)$ in Eq. (19), we obtain the characteristic equation for the eigenvalues $\bar{\Lambda}$ of the comparison system (19)-(20):

$$
\lambda \pm i \omega=\bar{\Lambda}+K\left(1-\frac{\sinh (\bar{\Lambda} \varepsilon)}{\bar{\Lambda} \varepsilon} e^{-\bar{\Lambda} T_{0}}\right) .
$$

Under the conditions of the MAN theorem, the stability of the original system (17)-(18) is determined by the roots $\bar{\Lambda}$ of the characteristic equation (21), providing that the frequency $a$ of variation of the delay is large compared to the system's dynamics. In this sense, the roots $\bar{\Lambda}$ may be considered as effective eigenvalues describing the overall stability of the original variable-delay system.

The presence of the parameters $\varepsilon$ and $T_{0}$ determining the delay interval makes the characteristic equation (21) transcendental in $\bar{\Lambda}$, possessing countable infinite set of complex solutions. The control is successful if for some $\varepsilon, K$ and $T_{0}$ the real parts of all the eigenvalues $\bar{\Lambda}$ are negative.

An alternative heuristic way to arrive to Eq. (21) is the following. We shall look for a solution of Eq. (18) in the form $\mathbf{x}(t) \sim \exp (\Lambda(t) t)$. The equation for $\Lambda(t)$ is

$\dot{\Lambda} t+\Lambda=\lambda \pm i \omega+K\left(e^{[\Lambda(t-T(t))-\Lambda(t))] t-\Lambda(t-T(t)) T(t)}-1\right)$.

Considering the asymptotic domain $t \gg 1$ (and therefore $t \gg T(t))$, the finite variation of the delay-time $T(t)$ and the approximation $\Lambda(t-T(t))-\Lambda(t) \approx-\dot{\Lambda}(t) T(t)$, from Eq. (22), one can conclude that there is an asymptotic relationship $\dot{\Lambda}(t) \sim 1 / t$, implying that asymptotically $\Lambda(t)$ becomes a constant. Integrating (22) over a period of the time delay function $T(t)$ in the asymptotic domain of large $t$ results in Eq. (21).

We numerically analyze Eq. (21) to obtain the domains of stability of the fixed point in the parameter plane $K-$ $T_{0}$ for different values of $\varepsilon$, keeping the parameters of the unstable focus fixed at $\lambda=0.5$ and $\omega=\pi$. The results are shown in panels (a) through (d) of Fig. 3, corresponding to $\varepsilon=0,0.3,0.5$ and 1 respectively.

The shaded areas correspond to the set of control parameters $\left(K, T_{0}\right)$ for which the largest real part of the complex eigenvalues $\bar{\Lambda}$ is negative, indicating a successful control. For combinations $\left(K, T_{0}\right)$ belonging to the white area, $\max [\operatorname{Re}(\bar{\Lambda})]>0$, and the control is not possible. The values of $\max [\operatorname{Re}(\bar{\Lambda})]$ are given by the grayscale in the upper right corner of Fig. 3. The control becomes more robust for larger magnitude of the negative $\max [\operatorname{Re}(\bar{\Lambda})]$. When $\varepsilon=0$, the VDFC method reduces to the standard TDAS control method, and the domain of successful control is indicated in panel (a) of Fig. 3 [11]. As $\varepsilon$ becomes larger than zero and closer to 1 (panels (b) through $(d)$ ), the domain of control is drastically enlarged, reaching its maximum at $\varepsilon=1$ (panel $(d)$ ). We note that due to the limitation of the control parameter $\varepsilon$ to the interval $0 \leq \varepsilon \leq T_{0}$, the origin of the axis $T_{0}$ is taken at $T_{0}=\varepsilon$.

In panels (e) through (h) of Fig. (3) we show the stability regions corresponding to panels (a) through (d), 

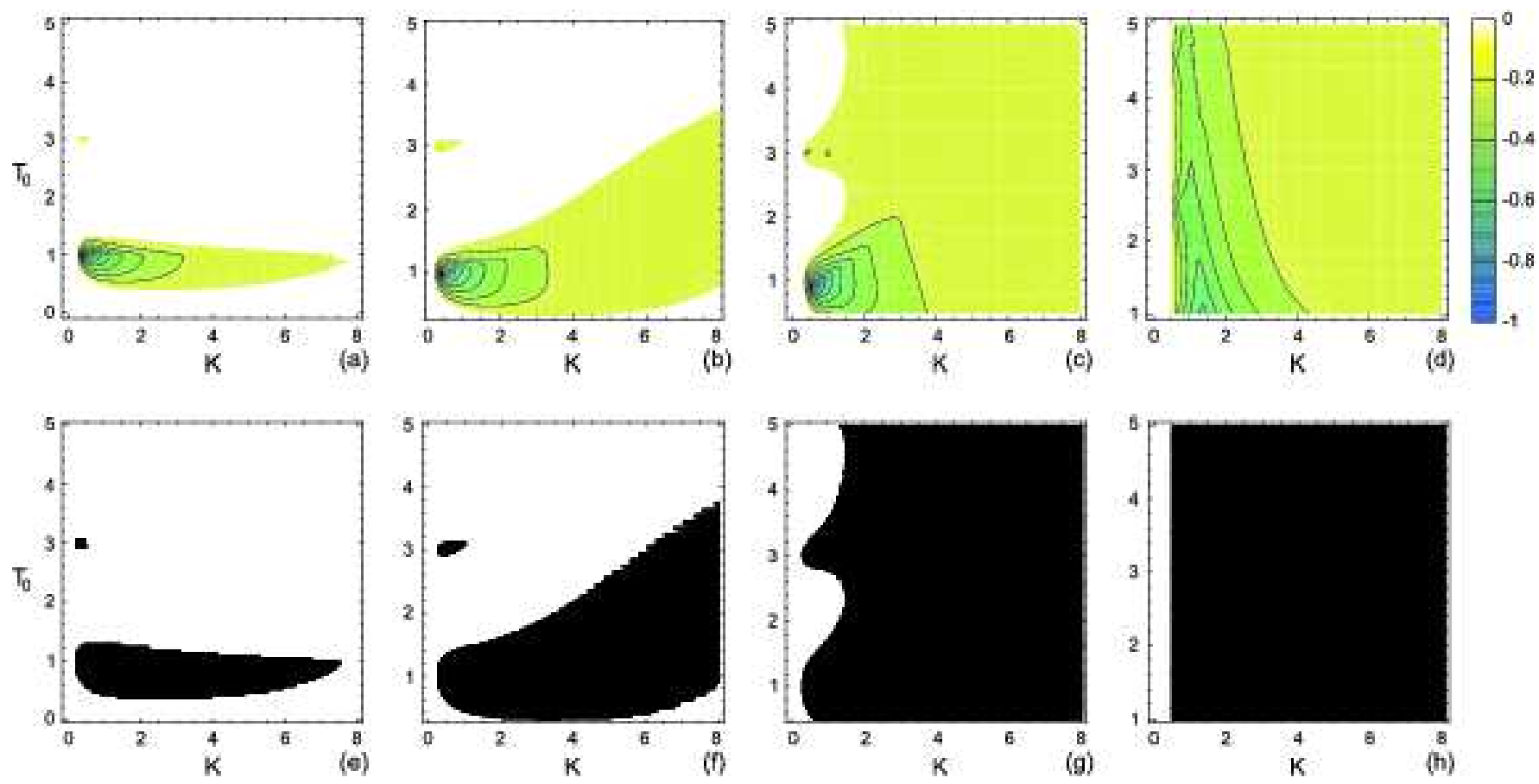

FIG. 3: (Color online) (a)-(d) Domains of control in the $\left(K, T_{0}\right)$ plane for a sawtooth-wave modulation of the delay time, obtained by a numerical solution of the characteristic equation (21). The values of the modulation amplitude are: (a) $\varepsilon=0$, (b) $\varepsilon=0.3$, (c) $\varepsilon=0.5$, (d) $\varepsilon=1$. For combinations of $K$ and $T_{0}$ belonging to the shaded areas, the largest real part of the complex eigenvalues $\bar{\Lambda}$ is negative, and the control is successful. The parameters of the unstable focus are $\lambda=0.5$ and $\omega=\pi$. (e)-(h) The corresponding stability regions (black areas) determined by a computer simulation of VDFC for the system (18). Note the shifts of the origin along the $T_{0}$-axis by an amount equal to $\varepsilon$.

obtained by a computer simulation of the VDFC control scheme (18). The simulation is performed by picking a point from the parameter plane $K-T_{0}$ and numerically integrating the system (18). The time-modulation of $T(t)$ used in the simulation was in a form of a sawtooth wave (8) with an inverse period $a=10$. The combinations $\left(K, T_{0}\right)$ that lead to a successful fixed point stabilization are marked in black. We immediately notice an excellent resemblance between the stability domains obtained by computer simulation and by numerically analyzing the characteristic equation (21). The resemblance may be further improved by requiring a larger accuracy of the Runge-Kutta method used in the simulation.

To obtain an analytical description of the boundaries of the control domain in Fig. 3, we substitute $\bar{\Lambda}=\Pi+$ $i \Omega$ into the characteristic equation (21) and separate the equation into real and imaginary parts. Since $\Pi=0$ at the threshold of control, the resulting equations are reduced to:

$$
\begin{aligned}
\lambda & =K\left(1-\frac{\sin (\Omega \varepsilon)}{\Omega \varepsilon} \cos \left(\Omega T_{0}\right)\right), \\
\pm \omega & =\Omega+K \frac{\sin (\Omega \varepsilon)}{\Omega \varepsilon} \sin \left(\Omega T_{0}\right),
\end{aligned}
$$

which can be algebraically manipulated to obtain a parametric representation of the dependence on $\Omega$ of the boundary of the successful control domain

$$
\begin{aligned}
K(\Omega) & =\frac{\lambda \pm \sqrt{\lambda^{2}-\left\{1-[\sin (\Omega \varepsilon) /(\Omega \varepsilon)]^{2}\right\}\left[\lambda^{2}+(\omega-\Omega)^{2}\right]}}{1-[\sin (\Omega \varepsilon) /(\Omega \varepsilon)]^{2}}, \\
T_{0}(\Omega)_{1} & =\frac{1}{\Omega}\left[2 n \pi+\arccos \left(\frac{\lambda[\sin (\Omega \varepsilon) /(\Omega \varepsilon)]^{2} \pm \sqrt{\lambda^{2}-\left\{1-[\sin (\Omega \varepsilon) /(\Omega \varepsilon)]^{2}\right\}\left[\lambda^{2}+(\omega-\Omega)^{2}\right]}}{[\sin (\Omega \varepsilon) /(\Omega \varepsilon)]\left[\lambda \pm \sqrt{\lambda^{2}-\left\{1-[\sin (\Omega \varepsilon) /(\Omega \varepsilon)]^{2}\right\}\left[\lambda^{2}+(\omega-\Omega)^{2}\right]}\right]}\right)\right], \\
T_{0}(\Omega)_{2} & =\frac{1}{\Omega}\left[2(n+1) \pi-\arccos \left(\frac{\lambda[\sin (\Omega \varepsilon) /(\Omega \varepsilon)]^{2} \pm \sqrt{\lambda^{2}-\left\{1-[\sin (\Omega \varepsilon) /(\Omega \varepsilon)]^{2}\right\}\left[\lambda^{2}+(\omega-\Omega)^{2}\right]}}{[\sin (\Omega \varepsilon) /(\Omega \varepsilon)]\left[\lambda \pm \sqrt{\lambda^{2}-\left\{1-[\sin (\Omega \varepsilon) /(\Omega \varepsilon)]^{2}\right\}\left[\lambda^{2}+(\omega-\Omega)^{2}\right]}\right.}\right)\right],
\end{aligned}
$$


where $n$ is a nonnegative integer characterizing different leaves arising from the multivaluedness of the arccosine function.

When $\varepsilon=0$ [see panels (a) and (e) in Fig. 3], VDFC is reduced to TDAS, and Eqs. (23) and (24) read [11]:

$$
\begin{aligned}
\lambda & =K\left(1-\cos \left(\Omega T_{0}\right)\right), \\
\pm \omega & =\Omega+K \sin \left(\Omega T_{0}\right) .
\end{aligned}
$$

In this case, when the product $\Omega T_{0}$ is an odd multiple of $\pi$, we obtain $\Omega=\omega$ and $K=K_{\min }=\lambda / 2$. Hence, the points $K=K_{\min }=\lambda / 2$ and $T_{0}=(2 n+1) \pi / \omega$ correspond to points of successful control in the $K-T_{0}$ plane with minimal feedback gain. Similarly, when $\Omega T_{0}$ is an even multiple of $\pi$, that is, when $T_{0}=2 n \pi / \omega$, the control fails for any feedback gain. The domain of control consists of stability islands at $T_{0}$ corresponding to odd $n$ isolated by regions encompassing $T_{0}$ corresponding to even $n$ for which the control fails.

When $\varepsilon>0$ [panels (b)-(d) and (f)-(h) in Fig. 3], from Eqs. (23) and (24) we obtain:

$$
K=K_{\min }=\frac{\lambda}{1+\sin (\omega \varepsilon) /(\omega \varepsilon)}
$$

for $\Omega T_{0}=(2 n+1) \pi$, and:

$$
K=K_{\min }=\frac{\lambda}{1-\sin (\omega \varepsilon) /(\omega \varepsilon)}
$$

for $\Omega T_{0}=2 n \pi$, implying considerable reconfiguration of the stability islands as soon as $\varepsilon>0$, which is readily observable from Fig. 3. Specifically, if $\varepsilon=1$ and $\omega=\pi$, then $K_{\text {min }}=\lambda$ for any integer $T_{0}$, as is clearly indicated from panels (d) and (h) in Fig. 3.

To conclude this section, we numerically calculated the parameter regions of the effective fixed point control for the Lorenz system (5)-(7) and the Rössler system (11)(13) in the case of a periodic sawtooth-wave modulation (8). The calculations were performed for different values of the modulation amplitude $\varepsilon$, following the same strategy as for the two-dimensional case discussed previously. We omit showing the resulting characteristic equations and the parametric formulas describing the boundary of the domain of control due to their length and complexity. The obtained stability regions are depicted in Fig. (4).

\section{CONCLUSION}

We have demonstrated that implementation of a timevarying delay into the standard TDAS control scheme can dramatically improve the efficiency of control of unstable steady states. The method of variable delay feedback control (VDFC) was illustrated for the paradigmatic Lorenz and Rössler systems, showing the usual control failure of TDAS in the case of unstable steady states having an odd-number of positive real eigenvalues. We expect that this limitation may be prevailed by a suitable choice of the feedback control matrix $K$ 22, 25, 26], or by using the VDFC method in combination with more sophisticated control schemes [27, 28].

Using Michiels-Van Assche-Niculescu theorem 24 and an independent approach for the case of a time-varying delay in a form of a sawtooth-wave, we were able to perform a linear stability analysis to calculate the domain of control for a two-dimensional unstable focus in the plane parametrized by the feedback gain $K$ and the nominal time-delay $T_{0}$ for different values of the parameter $\varepsilon$ determining the amplitude of the modulation. The same analysis was repeated for the three-dimensional Lorenz and Rössler systems. In parallel to the analytic derivation, we performed computer simulations that confirmed the results of the analytical approach. In this way, we showed that variable delay feedback control allows stabilization of unstable steady states over much larger domain of parameters in comparison to the usual TDAS control scheme.

Various modifications and extensions of the VDFC method, like using different forms of delay modulations, including a variable time-delay into the ETDAS scheme and other schemes, the influence of the nonzero controlloop latency [11], bandpass filtering and non-diagonal coupling, as well as experimental implementation of the VDFC, are all subjects of ongoing analysis.
[1] E. Ott, C. Grebogy and J. A. Yorke, Phys. Rev. Lett. 64, 1196 (1990).

[2] H. G. Schuster (ed.), Handbook of chaos control (WileyVCH, Weinheim, 1999).

[3] S. Boccaletti, C. Grebogi, Y. C. Lai, H. Mancini and D. Maza, Phys. Rep. 329, 103 (2000).

[4] T. Kapitaniak (ed.), Controlling Chaos (Academic Press, London, 1996).

[5] F. T. Arecchi, S. Boccaletti, M. Ciofini and R. Meucci, Int. J. Bifur. Chaos 8, 1643 (1998).

[6] K. Pyragas, Phys. Lett. A 170, 421 (1992).

[7] K. Pyragas and A. Tamaševičius, Phys. Lett. A 180, 99 (1993).
[8] K. Pyragas, Phil. Trans. R. Soc. A 364, 2309 (2006).

[9] J. E. S. Socolar, D. W. Sukow and D. J. Gauthier, Phys. Rev E 50, 3245 (1994)

[10] K. Pyragas, Phys. Lett. A 206, 323 (1995).

[11] P. Hövel and E. Schöll, Phys. Rev. E 72, 046203 (2005).

[12] S. Yanchuk, M. Wolfrum, P. Hövel and E. Schöll, Phys. Rev. E 74, 026201 (2006).

[13] T. Dahms, P. Hövel and E. Schöll, preprint, arXiv:0708.0147 (2007).

[14] K. Pyragas, V. Pyragas, I. Z. Kiss and J. L. Hudson, Phys. Rev. Lett. 89, 244103 (2002).

[15] K. Pyragas, V. Pyragas, I. Z. Kiss and J. L. Hudson, Phys. Rev. E 70, 026215 (2004). 

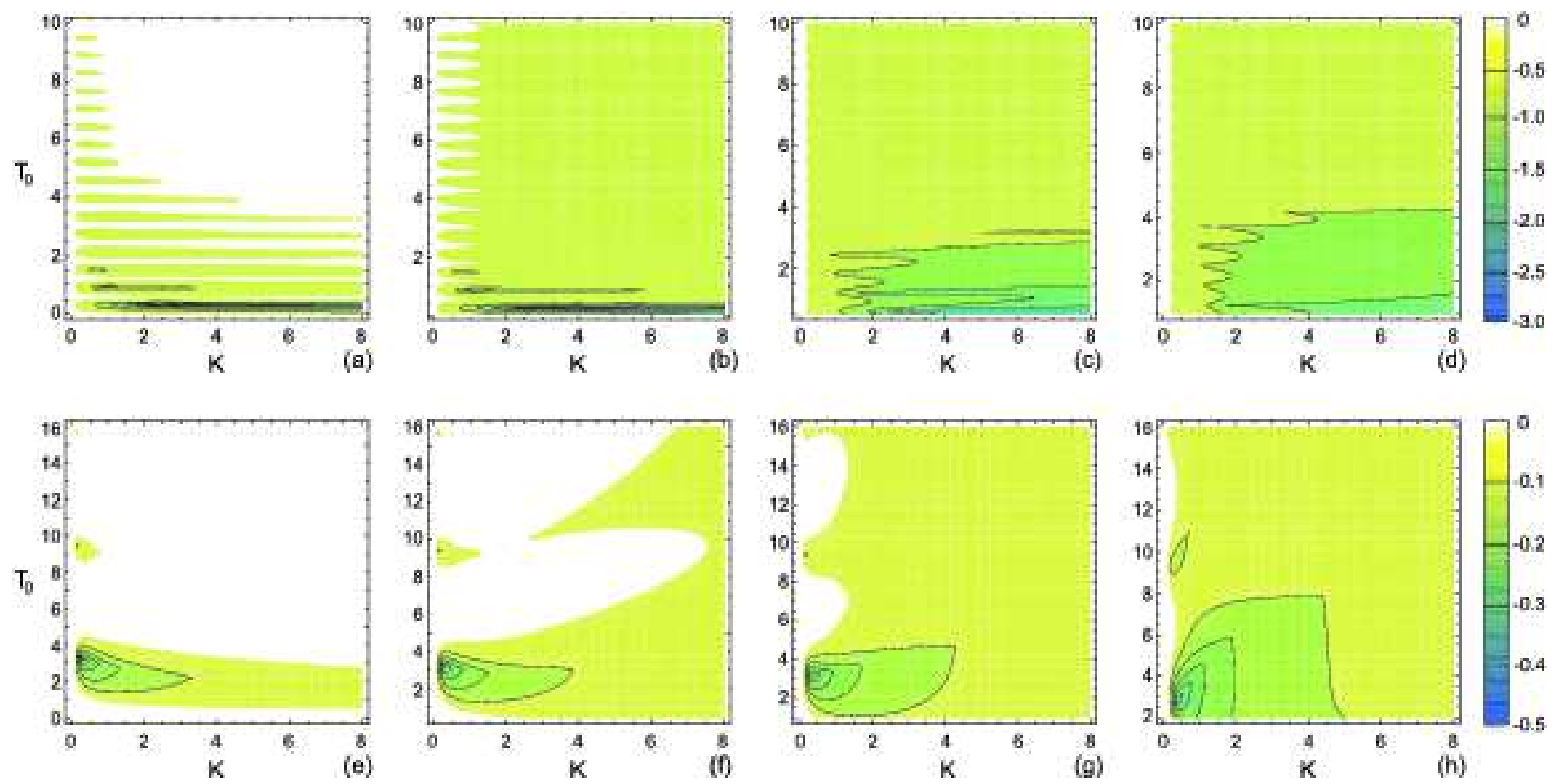

FIG. 4: (Color online) (a)-(d) Domains of successful control in the $\left(K, T_{0}\right)$ plane for the unstable steady states $C^{ \pm}$in the Lorenz system (5) - (17). The modulation of the delay time is in a form of a sawtooth-wave (8), and the values of the modulation amplitude are: (a) $\varepsilon=0$, (b) $\varepsilon=0.1$, (c) $\varepsilon=0.5$, (d) $\varepsilon=1$. (e)-(h) The corresponding domains of control for the unstable fixed point $C_{1}$ in the Rössler system (11)-(13). In this case, the values of the modulation amplitude are: (e) $\varepsilon=0,(\mathrm{f}) \varepsilon=0.5$, (g) $\varepsilon=1$, (h) $\varepsilon=2$. We note that the numerical calculations for the remaining two unstable states in the Lorenz and Rössler systems show no regions of successful control, in accordance with our previous discussion related to the odd-number limitation theorem.

[16] A. Albhorn and U. Parlitz, Phys. Rev. Lett. 93, 264101 (2004).

[17] A. Albhorn and U. Parlitz, Phys. Rev. E 72, 016206 (2005).

[18] E. N. Lorenz, J. Atmos. Sci. 20, 130 (1963).

[19] W. Just, T. Bernard, M. Ostheimer, E. Reibold and H. Benner, Phys. Rev. Lett. 78, 203 (1997).

[20] H. Nakajima, Phys. Lett. A 232, 207 (1997).

[21] H. Nakajima and Y. Ueda, Physica D 111, 143 (1998).

[22] B. Fiedler, V. Flunkert, M. Georgi, P. Hövel and E. Schöll, Phys. Rev. Lett 98, 114101 (2007).
[23] O. E. Rössler, Phys. Lett. A 57, 397 (1976).

[24] W. Michiels, V. Van Aasche and S. Niculescu, IEEE Trans. Autom. Control 50, 493 (2005).

[25] W. Just, B. Fiedler, M. Georgi, V. Flunkert, P. Hövel and E. Schöll, Phys. Rev. E 76, 026210 (2007).

[26] C. M. Postlethwaite and M. Silber, Phys. Rev. E 76, 056214 (2007).

[27] H. G. Schuster and M. B. Stemmler, Phys. Rev. E 56, 6410 (1997).

[28] K. Pyragas, Phys. Rev. Lett. 86, 2265 (2001). 